Secretary of IUPAC, Dr. M. Williams, who retired in 1997.

V. N. Parmon, Boreskov Institute of Catalysis, Siberian Branch of the Russian Academy of Sciences, Novosibirsk, Russia

H. Tributsch, Freie Universitat Berlin and HahnMeitner Institut, Dept. Solare Energetik, Berlin, Germany

A. V. Bridgwater, Bio-Energy Research Group, Chemical Engineering, Aston University, Birmingham, England, UK

D. O. Hall, Life Sciences Division, King's College, London, England, UK

\section{New Publications from the World Health Organization}

\section{Methyl tertiary-Butyl Ether, Environmental Health Criteria No. 206}

1998, xix + 199 pages (English with summaries in French and Spanish), ISBN 924157206 X, CHF 42.-/ USD 37.80; In developing countries: CHF 29.40, Order no. 1160206. WHO Distribution and Sales, CH1211 Geneva 27, Switzerland; E-mail: Publications@who.ch; Tel.: +41 2279124 76; Fax: +41227914857.

This book evaluates the risks to human health and the environment posed by exposure to methyl tertiarybutyl ether (MTBE). Used almost exclusively as a fuel additive, MTBE is blended with gasoline to provide both octane enhancement and an increase in oxygen content. MTBE currently numbers among the 50 chemicals produced in the highest volume worldwide. Production and use are expected to increase, particularly in countries where oxygenated or reformulated gasoline is required in national programs aimed at reducing ambient air levels of carbon monoxide and ozone or benzene and other volatile hydrocarbons.

A summary of sources of human and environmental exposure is followed by an assessment of what is known about the chemical's environmental behavior and fate. Studies demonstrate that, after discharge into air, MTBE largely remains in the air, with smaller amounts entering soil and water. Although atmospheric MTBE can partition into rain, data indicate that atmospheric formation by hydroxyl radicals is a more important pathway of removal.

Concerning environmental levels and human exposure, evaporative emissions from oxygenated gasoline are identified as the main source of exposure for the general population. Widespread exposure via inhalation is noted to occur during time spent at service stations, while driving cars, in public parking garages, and in homes with attached garages. The report also cites data on exposure levels obtained in numerous studies of urban air, in facilities where MTBE is manufactured or blended, and in such occupational settings as service stations, garages, and the transportation of neat MTBE and fuel mixtures through pipelines, barges, railroad cars, and trucks.

A review of the kinetics and metabolism of MTBE draws on toxicokinetic data derived from controlled studies in healthy adult volunteers and in occupationally exposed workers. Data indicate that MTBE is rapidly absorbed into the circulation following inhalation. In rodents, the compound is likewise rapidly absorbed and distributed following exposure by both inhalation and oral routes.

From studies in laboratory animals, the report identifies the principal signs of intoxication as depression of the central nervous system, ataxia, and labored respiration. Most effects on the central nervous system are transient. From the few studies available, the report concludes that MTBE is moderately irritating to the skin and eyes and induces slight to severe respiratory irritation. Repeated exposure results primarily in increases in organ weights and histopathological effects in the kidneys of rats and the liver of mice. The report found no evidence of adverse effects on reproduction, genotoxicity, or mutagenicity. Although limited carcinogenicity studies showed significant increases in tumor incidence, the report judged these findings inconclusive and thus inadequate to support an assessment of carcinogenic risk in humans.

An evaluation of health effects in humans gives major attention to several recent "outbreaks" of health complaints in the United States that occurred shortly after the introduction of MTBE-blended gasoline. Symptoms most widely reported by consumers include headache, eye and nose irritation, cough, nausea, dizziness, and disorientation. The report also draws on findings from epidemiological studies of occupationally exposed workers and from experimental studies of volunteers exposed in inhalation chambers. Based on this evaluation, the report concludes that MTBE alone, under common conditions of inhalation exposure, is not likely to induce acute adverse health effects in the general population. In making this conclusion, the report also notes that the potential effects of mixtures of gasoline and MTBE, as well as the manner in which most people are exposed to MTBE via oxygenated fuels, have not been examined experimentally or in prospective epidemiological studies. 


\section{Acetone, Environmental Health Criteria No. 207}

1998, xviii + 159 pages (English with summaries in French and Spanish), ISBN 924157207 8, CHF 36.-/ USD 32.40; In developing countries: CHF 25.20, Order no. 1160207 .

This book evaluates the risks to human health and the environment posed by exposure to acetone. Acetone is widely used as an intermediate in chemical production and as a solvent for resins, paints, inks, varnishes and lacquers, and in adhesives, thinners, and cleanup products. Pharmaceutical applications include use as an intermediate and solvent for drugs, vitamins, and cosmetics. Acetone is also used in food processing as an extraction solvent for oils and fats and as a precipitation agent in the purification of starches and sugars.

A discussion of sources of human and environmental exposure covers both natural and anthropogenic sources. In the mammalian body, studies show that acetone is formed endogenously from fatty acid oxidation, is found as a natural metabolic component in blood, urine, and human breath, and is exhaled. Acetone has been detected in a variety of plants and foods and is emitted, in vapor form, from several tree species. Acetone also occurs naturally as a biodegradation product of sewage, solid wastes, and alcohols, and as an oxidation product of humic substances. Concerning man-made sources of emissions, the report identifies the most important sources in wastewater discharges from many industries, leaching from industrial and municipal landfills, and evaporation of acetone solvent from coating products such as paints, cleaners, varnishes, and inks. Acetone may also be emitted from the combustion of wood and in exhaust from automobile, diesel, and turbine engines.

A review of data on environmental behavior and fate cites evidence from several studies of acetone levels detected in air, water, soil, and biological analyses. Studies indicate that atmospheric acetone is degraded by a combination of photolyis and reaction with hydroxyl radicals. Acetone is readily biodegradable in soil and water. A discussion of kinetics and metabolism draws on extensive data from absorption and tissue distribution studies, radiolabeled metabolic and kinetic studies, and studies of elimination and excretion. Abundant evidence shows that acetone is rapidly absorbed via the respiratory and gastrointestinal tracts and mainly distributed to non-adipose tissues. Under normal circumstances, metabolism is the predominant route of elimination. Studies in humans confirm the importance of such variables as diet, exercise, and alcohol consumption as factors affecting kinetics.

A review of toxicity studies in laboratory mammals and in vitro test systems concludes that acetone is only mildly toxic to the liver, unless physiological processes are compromised, as in diabetes mellitus. Some adverse effects on development and reproductive function have been reported. One of the major adverse effects identified is acetone's ability to potentiate the toxicity of other chemicals. Extensive studies on the mechanisms of toxic action help elucidate the possible mechanisms by which acetone enhances the neurotoxicity of ethanol.

A section on health effects in humans evaluates findings from numerous case reports of accidental or intentional poisoning, studies conducted in healthy volunteers, and studies of occupationally exposed workers. The most commonly reported effects include irritation to the nose, eyes, throat, and trachea. Studies also show that acetone can produce neurobehavioral and other changes, including headache, dizziness, confusion and, at high vapor concentrations, central nervous system depression and narcosis. The report found no evidence that acetone is either a skin or a respiratory tract sensitizer. Human studies confirm the ability of acetone to potentiate, and in some instances antagonize, the toxic effects of other chemicals. Those at greatest risk include diabetics, alcoholics, and those undergoing prolonged fasting. The report found no evidence that acetone is genotoxic or carcinogenic.

Concerning effects on the environment, the report concludes that acetone, even in the case of accidental spills, is unlikely to have a major or lasting adverse effect on the ecosystem.

\section{WHO Monographs on Selected Medicinal Plants, Volume 1}

1999, v + 289 pages (available in English; French in preparation), ISBN 924154517 8, CHF 92.-/USD 82.80; In developing countries: CHF 64.40, Order no. 1150460 .

This book provides a collection of 28 monographs covering the quality control and traditional and clinical uses of selected medicinal plants. Plants were selected for inclusion on the basis of their widespread use, particularly in countries that rely heavily on medicinal plants to meet primary health care needs. Monographs are provided for a number of medicines traditionally used to treat such common complaints as diarrhea, constipation, headache, appetite loss, sleep disorders, fatigue, and mild respiratory, gastrointestinal, and skin disorders. Additional medical applications assessed range from the lipid-lowering potential of garlic powder preparations, through the possible antiplasmodial activity of Fructus Bruceae, to the role of curcumin in promoting peptic ulcer healing and reducing the associated abdominal pain.

In preparing and publishing these monographs, WHO aims to encourage standardized scientific approaches to ensuring the safety, quality, and efficacy of medicinal plants and their products. The monographs are also intended to promote international harmoniza- 
tion in the quality control and use of herbal medicines and to serve as models for the development of national formularies. Draft monographs were finalized following review by over 100 experts in 40 countries. Some 1400 references to the literature are included.

Each monograph follows a standard format, with information presented in two parts. The first gives pharmacopoeial summaries for quality assurance, botanical features, distribution, identity tests, purity requirements, chemical assays, and active or major chemical constituents. A section on definition provides the Latin binomial pharmacopoeial name, the most important criterion in quality assurance. Latin pharmacopoeial synonyms and vernacular names, listed in the section on synonyms and selected vernacular names, are those names used in commerce or by local consumers.
The second part of each monograph begins with a list of dosage forms and of medicinal uses categorized as uses supported by clinical data, uses described in pharmacopoeias and in traditional systems of medicine, and uses described in folk medicine, but not yet supported by experimental or clinical data. Each monograph also includes an extensive review of available data on experimental and clinical pharmacology, followed by information on contraindications, such as sensitivity or allergy, warnings or precautions (particularly in such special groups as pregnant and breast-feeding women), adverse reactions, and dosage. A list of references concludes the monograph.

Additional medicinal plants will be covered in a second volume, which is currently undergoing review.

\section{Provisional Recommendations}

\section{IUPAC Seeks Your Comments}

In this section, we publish synopses of IUPAC's latest provisional recommendations on nomenclature and symbols. All comments on these recommendations are welcome and will be taken into consideration. The final revised versions are published in Pure and Applied Chemistry, and synopses of these are published in Chemistry International as recent reports.

If you would like to comment on the provisional recommendations, please write to your nearest national/ regional center to request a copy of the full report. Copies are not available from the IUPAC Secretariat. The most recent list of national/regional centers appeared in Chemistry International 1997, 17, 141. This information is also available on the IUPAC web site: http:// www.iupac.org/.

Analytical Chemistry Division. Commission on Analytical Nomenclature-Nomenclature for X-Ray Emission Spectrochemical Analysis

This draft document contains the nomenclature and practices of X-ray spectrochemical analysis, which is practically carried out by X-ray emission spectroscopy (XES), especially by the X-ray fluorescence method. Quantitative analysis by XES has excellent repeatability. Reproducibility and accuracy of XES, however, depend strongly on specimen preparation and data analysis. Some 60 terms together with definitions are contained herein. These terms are followed by condensed descriptions of the conditions that should be observed in order to realize feasible analytical performance.
General comments refer to similarity and difference of terms used in X-ray analysis and in other optical methods. Terms related to material examined describe specimen preparation-related matters. Terms related to $\mathrm{X}$-ray generation deal with the mechanism of X-ray generation and X-ray sources. These also refer to Xray attenuation, which is important in quantitative analysis. Terms related to X-ray measurement cover spectrometer and detector systems. Terms related to X-ray data interpretation concern statistical description of $\mathrm{X}$ ray intensity measurement and various quantification methods. The analytical function method and the fundamental parameter method, both widely used, are described in a concise manner referring to an ASTM standard. This document is a revision of a part of spectrochemical analysis in the Orange Book (Nomenclature System for X-Ray Spectroscopy, IUPAC Recommendations 1991).

Comments by 30 April 2000 to Prof. Yohichi Gohshi, Deputy Director General, National Institute for Environmental Studies, 16-2 Onogawa, Tsukuba 305-0053, Japan. Tel.: +81-298-50-2301; Fax: +81-298-51-2854; E-mail: gohshi@nies.go.jp.

Macromolecular Division. Commission on Macro-
molecular Nomenclature-Nomenclature of Regu-
lar Single-Strand Organic Macromolecules
A structure-based nomenclature for regular single-
strand organic polymers is described. In concept, a ge-
neric name for the polymer (ABC) is poly(ABC), in
which (ABC) is a constitutional repeating unit (CRU)
representing the chemical structure of the polymer
chain, and $\mathrm{A}, \mathrm{B}$, and $\mathrm{C}$ are the subunits that comprise 